\title{
Mutual coupling reduction in circularly polarized dielectric resonator MIMO antenna
}

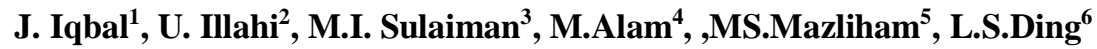 \\ ${ }^{1,2,3}$ Universiti Kuala Lumpur, British Malaysian Institute, batu 8, Jalan Sungai Pusu, 53100, Malaysia \\ ${ }^{1,2}$ Gomal University, Dera Ismail Khan, KPK, Pakistan \\ ${ }^{4}$ ILMA University, Korangi Creek, 74900, Pakistan \\ ${ }^{4}$ Universiti Kuala Lumpur, Malaysian Institute of Information Technology, 1016 Jalan Sultan Ismail, 50250, Malaysia \\ ${ }^{5}$ Universiti Kuala Lumpur, 1016 Jalan Sultan Ismail, 50250, Malaysia \\ ${ }^{6}$ RF Station Sdn.Bhd, A-2-7, Glomac Damansara No, 699, Taman Tun Dr Ismail, 60000, Malaysia
}

\begin{tabular}{l}
\hline Article Info \\
\hline Article history: \\
Received Dec 112018 \\
Revised Feb 15, 2019 \\
Accepted Feb 28, 2019 \\
\hline
\end{tabular}

\section{Keywords:}

Circular polarization

MIMO antenna

Mutual coupling

RDRA

\begin{abstract}
In this article a novel circularly polarized (CP) rectangular dielectric resonator antennas (RDRA's) array has been designed. A unique conformal feeding strip has been used to excite the RDRA's having a distance of $\lambda / 2$. Various techniques are investigated to reduce mutual coupling (MC). Different formations, comprise of reorientation of the antennas have been employed to suppress MC and to enhance $\mathrm{CP}$ bandwidth. By the unique orientation the mutual coupling has been reduced by $\sim 14 \mathrm{~dB}$. A broadband circular polarization over a bandwidth of $\sim 18.8 \%$ in conjunction with an impedance matching bandwidth of $\sim 11.3 \%$ has been achieved. The design has been modeled and simulated using computer simulation technology (CST). Two different simulation techniques i.e. finite integration technique (FIT) and Finite element method (FEM) have been used to compute and validate the results respectively. A significant resemblance in results have been observed.
\end{abstract}

Copyright $\odot 2019$ Institute of Advanced Engineering and Science. All rights reserved.

\section{Corresponding Author:}

\section{M.I. Sulaiman,}

Universiti Kuala Lumpur,

British Malaysian Institute,

Batu 8, Jalan Sungai Pusu, 53100Gombak, Malaysia.

Email: m.ismail.unikl.edu.my

\section{INTRODUCTION}

From las few decades' communication engineers are focusing more on DRAs because of there good features such as wide bandwidth, no metallic losses and flexible shapes [1-2]. Recently, a significant progress in wireless activities has boosted the multiple inputs multiple outputs (MIMO) antenna technologies. For wireless application, MIMO antenna systems are used for long-term evolution (LTE) network, wireless local area network (WLAN), and worldwide interoperability for microwave access (WiMAX). MIMO system offers better quality of services in non-line of sight (NLOS) communication since it offers significantly wider bandwidth of up to hundreds of gigabit compared to a single input single-output (SISO) system [3]. Additionally, MIMO antennas have the capability to improve the channel capacity over a limited available bandwidth to attain a high data rate. MIMO is an essential part of existing technologies because of enhancement of different parameters, i.e. gain, data rate, capacity, and efficiency, etc.

The concept of MIMO was initiated using the capacity theorem [4]. In wireless communication system, MIMO has been used in conjunction with pulse amplitude modulation (PAM) signals [5], directional digital transmission and reception using beam-forming signal-processing applications [6-7], digital transmission systems with multi-channel [8-9] and multivariate analysis with memory over the Gaussian channel [10]. In modern wireless application, most of the devices are compact sizes. The handy and small 
devices set a bound on the space between antennas in MIMO system. The close vicinity of the radiating antennas in MIMO can cause the degradation of different antenna parameters such as gain and return loss. Such degrading outcome is jointly known as a mutual coupling, which rigorously affects the near and far field characteristics. A low value of mutual coupling need to be maintained between the antennas to ensure an efficient MIMO antenna systems, [11].

MIMO system using microstrip patch antennas have low efficiency such as in [12], where a dualband patch antenna is used together with capacitive loaded loops. In such design, the antenna efficiency in lower and higher frequency band has about 30\% and $70 \%$ respectively. This shortcoming of low efficiency in multiple patch antenna systems can be solved by replacing the patch antennas with dielectric resonator antennas (DRAs) for MIMO since DRAs possess very high radiation efficiency [13].

A number of methods has been proposed by different researchers to decrease mutual coupling between DRAs. About $20 \mathrm{~dB}$ isolation has been achieved by using artificial magnetic conductor (AMC) ground plane [14]. In [15], a $24 \mathrm{~dB}$ reduction in mutual coupling has been obtained using EBG structure between radiating elements. A Split ring resonators in [16] reduces mutual coupling by approximately $28 \mathrm{~dB}$. Paper [17] proposed the use of an arc between two rectangular DRAs, which causes a $5 \mathrm{~dB}$ reduction in mutual coupling. On the other hand for exciting, conformal strip is used to excite the DRA as that is easier in use and provide better impedance matching with DRAs [18-19].

Previously, researcher focus a lot on linearly polarized antennas but due to the drawbacks of degradation of quality issue cause by the orientation of transmitter and receiver antennas [20], Now a day's high attention toward circular polarization has been paid, as circularly polarized antenna system is insensitive to the orientation as claimed in the literature [21-25].

\section{ARRAY GEOMETRY AND DESIGN}

Figure 1 illustrates the RDRA Configuration and dimension of conformal metallic strip to excite the antenna [18] i.e. $\mathrm{a}=26.1$ (height) $\mathrm{mm}, \mathrm{b}=25.4$ (breadth) $\mathrm{c}=14.3$ (width) and permittivity $(\varepsilon r)=8$. The novel feed is made up of five individually cut strips. The optimized feed parameters are h1=11 mm, h2=10.50 mm, $\mathrm{d} 1=10.5 \mathrm{~mm} \mathrm{~d} 2=1 \mathrm{~mm} \mathrm{~d} 3=2.5 \mathrm{~mm}$ and $1=8.0 \mathrm{~mm}$ respectively. Similarly for parasitic patch the dimension are same as in [26]. The boundary condition of $\mathrm{Zmin}$ is set to be $\mathrm{Et}=0$ in order to simulate the effect of an infinite ground plane. The Proposed feed has been exited through discrete edge port. Mashed parameters like, lines per wavelength, lower mesh limit and Mesh line ratio limit is 62, 37, and 52 respectively and finally, for the proposed final formation of Antenna array, mesh cells for the RDRA array without parasitic is 5, 10,439. Furthermore, same mashed parameters are kept for RDRA array with parasitic patch. The optimized design has been simulated by using hexahedrons meshing.

The novel design has been simulated in CST by using transient solver, which is based on the finite integration technique (FIT).The conformal metal strip feed has been used as it delivers better impedance matching with RDRA's.

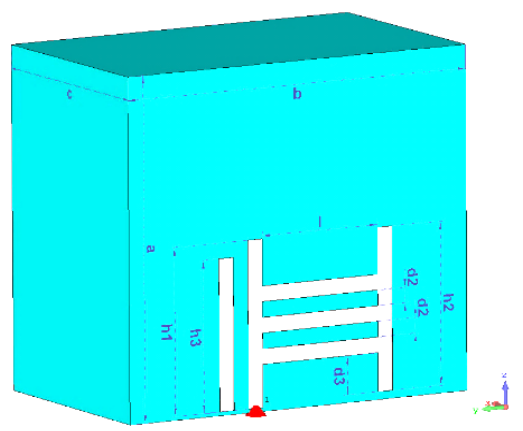

Figure 1. Configuration of the RDRA and conformal Strip

Above mentioned RDRA has been used in two antenna-system. The DRA's are placed back-to-back and the center-to-center spacing between the RDRA is chosen to be $35.62 \mathrm{~mm}$ that is equivalent to $\lambda 0 / 2$ at 4.2 $\mathrm{GHz}$ as demonstrated in Figure 2a.In order to examine the outcome of antenna orientation on antenna MC, two additional topologies have been studied in Figures $2 b$ and $2 c$ with approximately same center-tocenter spacing. In the orientation of Figure $2 \mathrm{~b}$, one of the DRA is rotated $90^{\circ}$ with respect to the other one making orthogonal MIMO array while on the other side the RDRA's of Figure $2 \mathrm{c}$ are aligned. 
In the last orientation the DRA's are placed face-to-face and the center-to-center spacing between the RDRA is chosen to be similar as above mentioned cases as demonstrated in Figure $2 \mathrm{~d}$.

\section{RESULTS AND DISCUSSIONS}

The basic reason for mutual coupling is either through propagating surface waves or by means of radiation [26], in order to overcome such issues different orientations have been examined that are discussed below in detail.
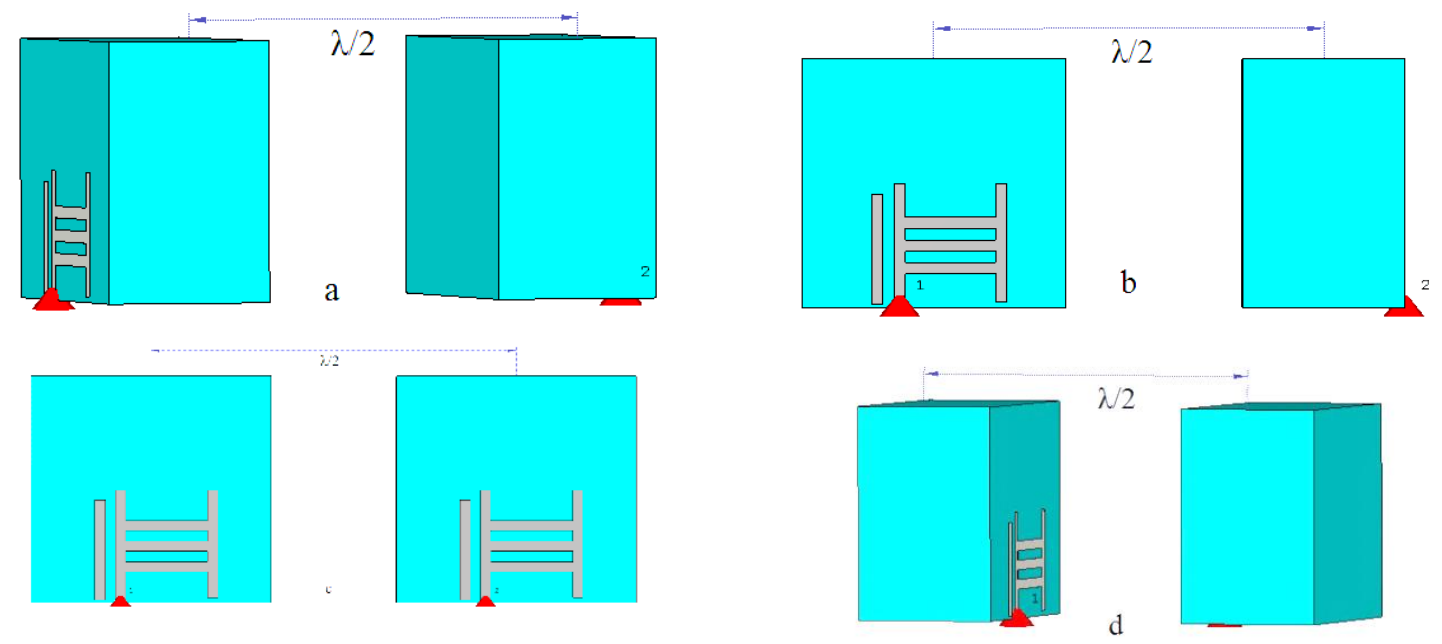

Figure 2. Two-antenna systems composed of RDRA's of Figure 1 with (a) Back-to-Back, (b) perpendicular, (c) aligned, and (d) Face-to-Face Orientations

\subsection{Back-to-Back Orientation of RDRA Array}

The simulated reflection coefficients $(\mathrm{S} 11<-10 \mathrm{~dB})$ have been presented in Figure 3, the impedance matching bandwidth of $9.06 \%$ have been achieved at resonance frequency (f0) of $4.24 \mathrm{GHz}$. In parallel $3 \%$ of $3 \mathrm{~dB}$ axial ratio bandwidth attained but at different frequency range. Hence the designed geometry is not circularly polarized.

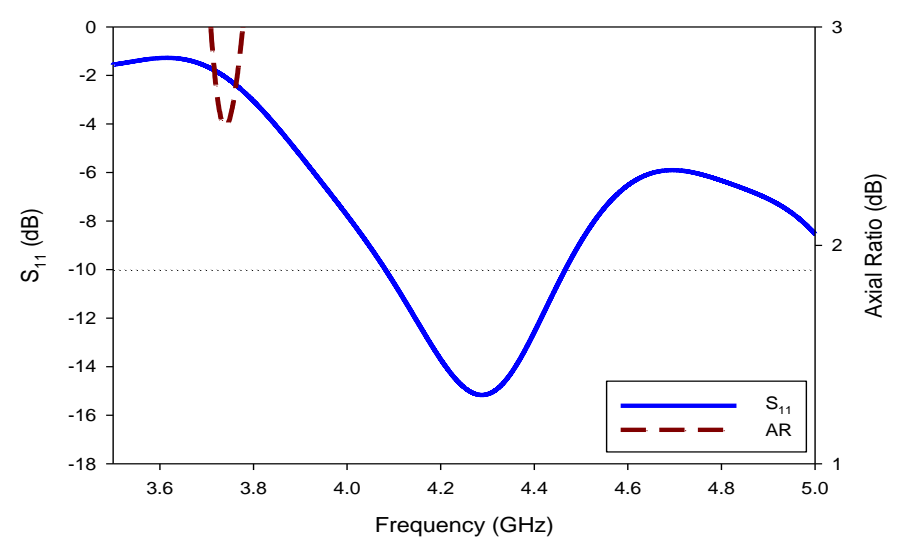

Figure 3. Return loss of back-to-back RDRA array with AR

\subsection{Orthogonal Orientation of RDRA Array}

As demonstrated in Figure 2b, one RDRA antennas is rotated $90^{\circ}$ with respect to the other one, making an orthogonal shape. Additionally, this Configuration helped in achieving the circular polarization. In 
Figure 3 the return losses and $3 \mathrm{~dB}$ axial Ratio of the perpendicularly placed RDRA's have been presented. The proposed formation offers the return loss $\mathrm{S} 11<-10 \mathrm{~dB}$ bandwidth of $\sim 11 \%$ and circular polarization bandwidth of $7.38 \%$ with a nominal overlap of $\sim 1.94 \%$. On the other side, as depicted in Figure 4 Mutual coupling S21 of $4.75 \mathrm{~dB}$ has been computed at the minimum of axial ratio i.e. $4.02 \mathrm{GHz}$. Isolation of perpendicular RDRA array as shown in Figure 5.

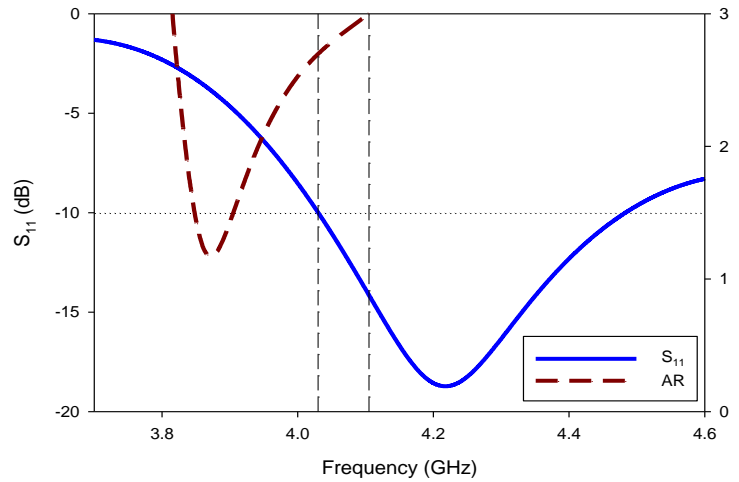

Figure 4. Return loss of perpendicular RDRA array with AR

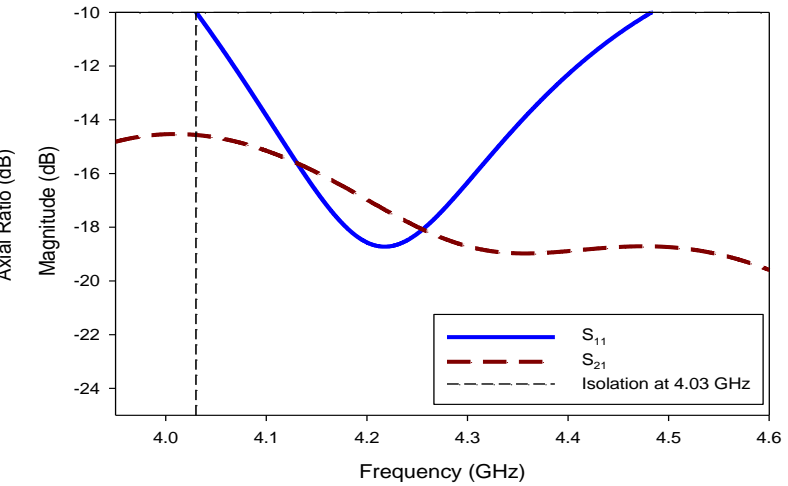

Figure 5. Isolation of perpendicular RDRA array

\subsection{Aligned Orientation of RDRA Array}

In this layout both RDRA's antennas are aligned as presented in Figure 2c, using such Configuration the CP bandwidth has been enhanced as well as isolation compared to the previous cases. Both the impedance matching bandwidth and $3 \mathrm{~dB}$ axial ratio of the aligned geometry has been demonstrated in Figure 6. The return loss improves around $6 \%$ to that of orthogonal geometry i.e.17.6\% additionally, circular polarization bandwidth improves to $11.2 \%$ with an overlap of $\sim 7 \%$.Similarly the mutual coupling S21 has been suppressed to $8 \mathrm{~dB}$, at the minimum of axial ratio as shown in Figure 7.

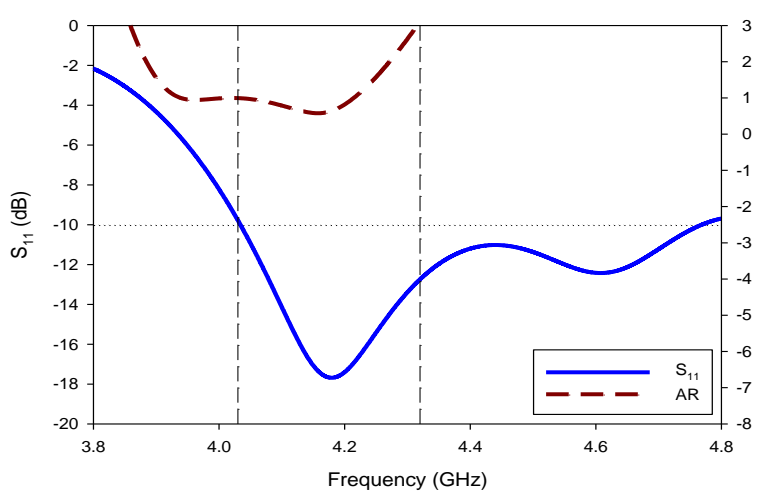

Figure 6. Return loss of aligned RDRA array with AR

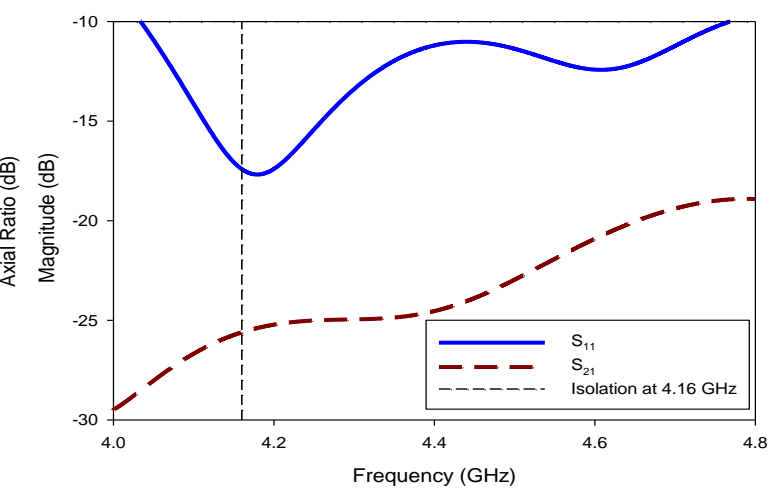

Figure 7. Isolation of aligned RDRA array

\subsection{Face-to-Face Orientation of RDRA Array}

The proposed array has been designed by placing the RDRA's in face-to-face conFigureuration as shown in Figure 2d. By using such conFigureuration a wideband circular polarization along with good impedance matching bandwidth has been achieved over same frequency range. Along with this mutual coupling has been suppressed considerably.

The Figure 8 represents the return losses and the AR bandwidth of the proposed geometry. A broadband of $3 \mathrm{~dB}$ axial Ratio bandwidth of $\sim 18.3 \%$ has been achieved. The minimum point of axial ratio has been computed at $4.47 \mathrm{GHz}$. An impedance matching bandwidth of $\sim 11 \%$ has been offered by the proposed design at resonant frequency of $4.24 \mathrm{GHz}$. The in conjunction overlapped bandwidth of $\sim 11 \%$ has been provided by the proposed geometry. The isolation between the antennas has been demonstrated in 
Figure 9. This conFigureuration offers the maximum mutual coupling suppression i.e. $\sim 14 \mathrm{~dB}$. All this parameters of the final designed has been optimized by using FIT in CST. The results of FIT have been validated using FEM i.e. another computational technique abatable in CST. A minor deviation in the bandwidth and resonant frequency obtained can be attributed to the different computational techniques. In overall, there is a reasonable trend resemblance between them.

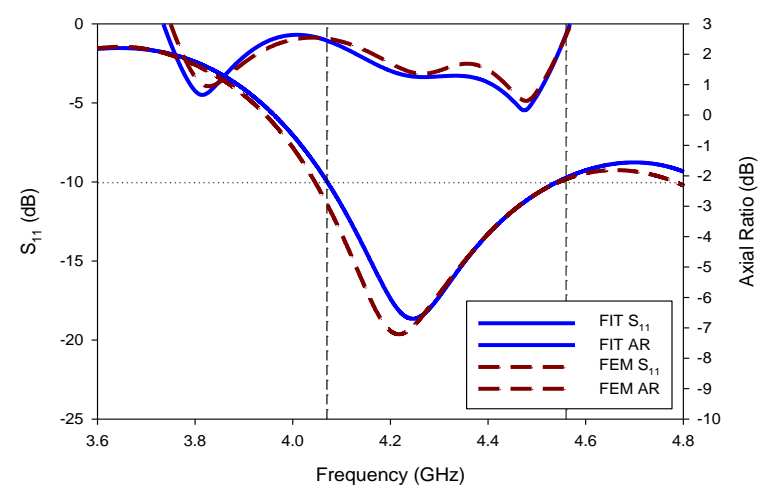

Figure 8. Return loss of face-to-face RDRA array with AR

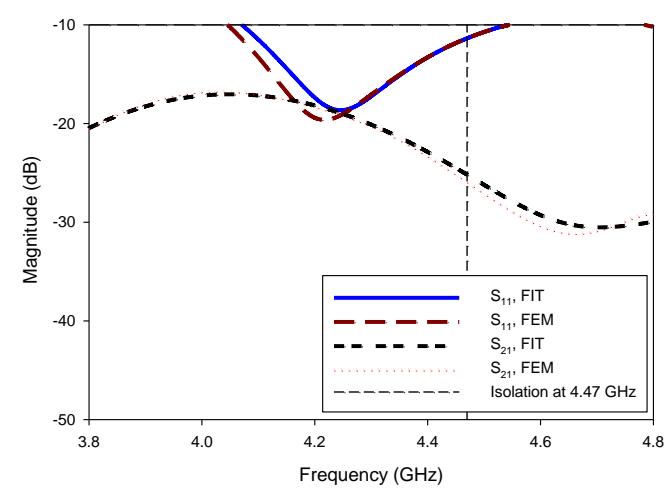

Figure 9. Isolation of face-to-faceRDRA array

Additionally, due to the reduction in mutual coupling the proposed orientation array offers a satisfactory simulated boresight gain of $\sim 5.95 \mathrm{dBi}$ as demonstrated in Figure 10.

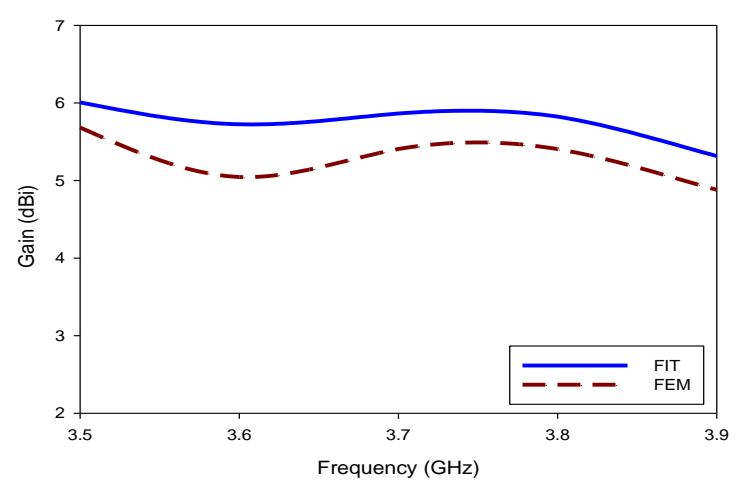

Figure 10. Gain of proposed orientation

In Figure 10 the current distribution of proposed antenna geometry is shown that is co-centric while Figure 11 demonstrate the current distribution of Figure 2a, the current direction is anti-clockwise which clearly shows that the direction of current changes which in result reduces MC basically, The spacing and geometry between the DRA's of the proposed design act in this way which prevent current inducing directly from the aggressor antenna to the victim antenna which in result change the current flow on the ground and that is the main reason of decrease the mutual coupling [19].

Higher the isolation and lower the envelope correlation coefficient (ECC) are important in MIMO systems [27].The ECC is in linked to the mutual coupling, and it is directly proportional to the mutual coupling between arrays. The ECC can be achieved using the far-field characteristics [28] of the radiating elements. Let the F1 $(\theta, \varphi)$ and F2 $(\theta, \varphi)$ are the field patterns of proposed RDRA array, then ECC is given below:

$$
\left|\rho_{e}(i, j, N)\right|=\frac{\left|\sum_{n=1}^{N} S_{i, n}^{*} S_{n, j}\right|}{\sqrt{\left|\prod_{k(=i, j)}\left[1-\sum_{n=1}^{N} S_{i, n}^{*} S_{n, k}\right]\right|}}
$$


In the same way, the diversity gain (DG) is also dependent upon mutual coupling [28], which is acquired in terms of maximum theoretical DG $(10 \mathrm{~dB})$ and $\rho$ by using equation given below. DG and correlation is inversely proportional to each other. From Figures 12-13 it is obvious that proposed RDRA array have lower ECC value and higher DG values i.e. $\sim 4.57 \mathrm{e}-007$ and $\sim 9.99 \mathrm{dBi}$ respectively.

$$
G_{D G}=10 \times \sqrt{1-|\rho|^{2}} .
$$

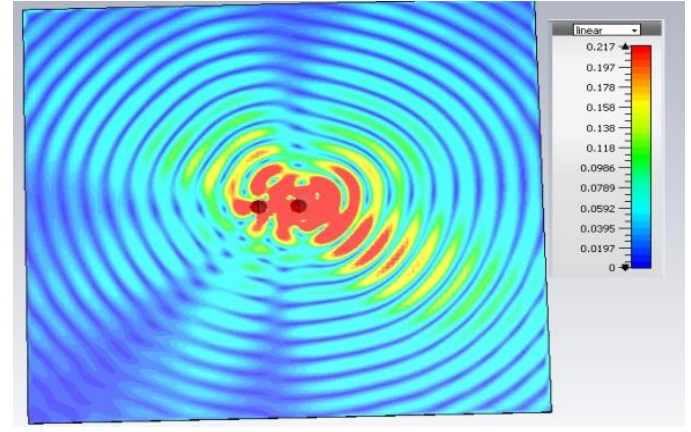

Figure 10. Current flow of face-to-face RDR Array

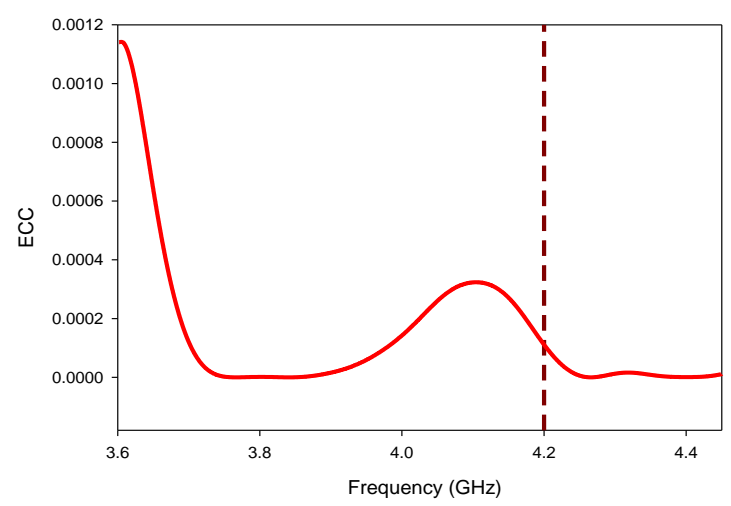

Figure 12. ECC of Proposed RDRA array

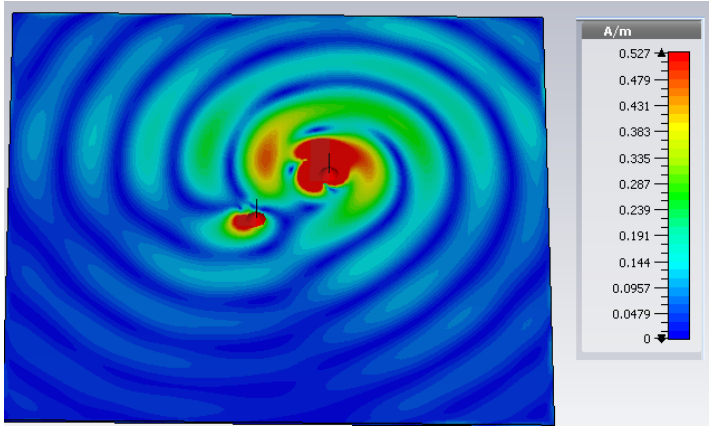

Figure 11. Current flow of back-to-back RDR Array

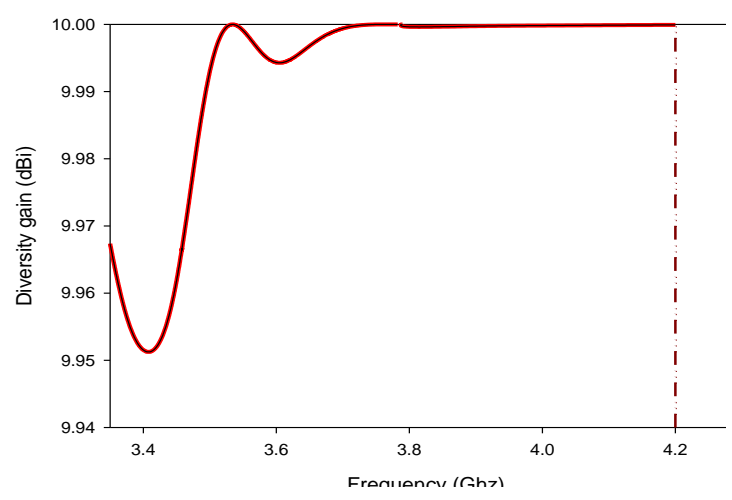

Figure 13. DG of proposed RDRA array

The radiation patterns simulated using FIT at $0^{\circ}$ and $90^{\circ}$ are shown in Figure 14. From these result, it is evident that the MIMO design generates a left hand circular polarized (LHCP) wave since the left-hand field component is stronger than the right-hand component.
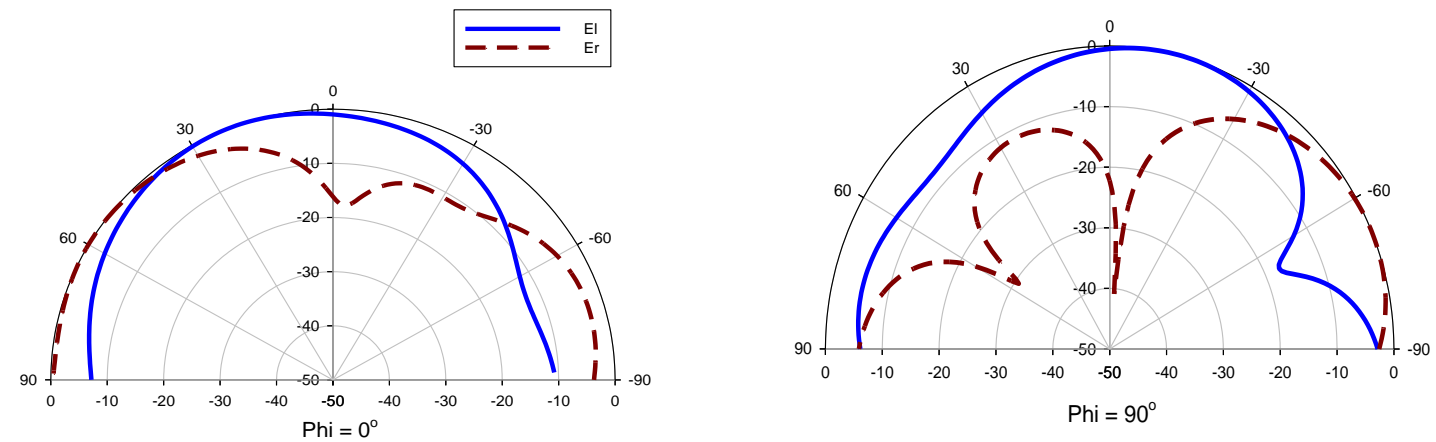

Figure 14. Radiation pattern of Slotted CDRA Array 


\section{CONCLUSION}

This paper proposed a two-element RDRA array with different formation to suppress MC and to enhance CP bandwidth. By the face-to-face orientation the mutual coupling has been reduce over a wideband frequency of 4.06-4.54 GHz. Additionally, an overlapping bandwidths of $\sim 7 \%$ has been achieved in which a return loss $\left(\mathrm{S}_{11}<-10 \mathrm{~dB}\right)$ is obtained in conjunction with a $3-\mathrm{dB}$ axial ratio Two different simulation techniques i.e. finite integration technique (FIT) and Finite element method (FEM) have been used to compute and validate the results respectively. A significant resemblance in results have been observed.

\section{ACKNOWLEDGMENT} my research.

I am very thankful to UniKL for awarding Short term research grant (STRG) to support

\section{REFERENCES}

[1] D. Subramaniam, M. Jusoh, T. Sabapathy, M.N. Osman, M.R. Kamarudin, M.T. Ali, M.N.M. Yasin, A. Ali, H.A. Majid, and R. Rahim, "A Stacked Planar Antenna with Switchable Small Grid Pixel Structure for Directive High Beam Steering Broadside Radiation," International Journal of Engineering and Technology (UAE), 7(2.5 Special Issue 5), 2018, pp.122-127.

[2] M. N. M. Yasin and S. K. Khamas, "Measurements and Analysis of a Probe-Fed Circularly Polarized Loop Antenna Printed on a Layered Dielectric Sphere," in IEEE Transactions on Antennas and Propagation, vol. 60, no. 4, , April 2012, pp. 2096-2100.

[3] Paulraj, A.; Kailath, T."Increasing Capacity in Wireless Broadcast Systems using Distributed Transmission/Directional Reception”. U. S. Patent No. 5345599, 1994.

[4] M. S. Sharawi, A. B. Numan and D. N. Aloi, "Isolation Improvement in A Dual-Band Dual-Element MIMO Antenna System using Capacitively Loaded Loops," Progress in Electromagnetics Research, Vol 134,: pp 247-266, 2013.

[5] Shannon, C.E. “A Mathematical Theory of Communication”. Bell Syst. Tech. J', vol 27, pp-379-423, 1948.

[6] Yang, D.G.; Kim, D.O.; Kim, C.Y.”Design of A Dual Band MIMO Monopole Antenna with High Isolation Using Slotted CSRR for WLAN" Microw. Opt. Technol. Lett.2014;, 56 (10) : 2252-2257.

[7] Singh, H.S.; Pandey, G.K.; Bharti, P.K.; Meshram, M.K.”A Compact Dual-Band Diversity Antenna for WLAN Applications with High Isolation”. Microw. Opt. Technol. Lett., vol 57 ,no 4,pp-906-912, 2015.

[8] Salz, J."Digital Transmission Over Cross Coupled Linear Channels". AT \& T Tech. J., vol 64,no 6, pp- 1147-1159, 1985.

[9] Branderburg, L.H.; Wyner, A.D. "Capacity of the Gaussian Channel with Memory" the multivariate case. Bell Syst. Technol. J., vol 53,no 5, pp- 745-778, 1974.

[10] Roy, R.; Bjorn, O."Spatial Division Multiple Access Wireless Communication Systems". U.S. Patent No. $5515378,1996$.

[11] M. S. Sharawi, A. B. Numan and D. N. Aloi, "Isolation Improvement in A Dual-Band Dual-Element MIMO Antenna System using Capacitively Loaded Loops," Progress in Electromagnetics Research, ; vol 134, pp- 247-266, 2013.

[12] Z. Guiping, A. A. Kishk, A. W. Glisson, and A. B. Yakovlev, "A Mutual Coupling Reduction Technique for Dielectric Resonator Antennas Over AMC Surface,” in Proc. IEEE AP-S Int. Symp., pp-377-380, Jul. 2006.

[13] M. J. Al-Hasan, T. A. Denidni and A. R. Sebak, "Millimeter-Wave Compact EBG Structure for Mutual-Coupling Reduction in Dielectric Resonator Antenna Arrays," in Proc. IEEE AP-S Int. Symp., pp 95-96, Jul. 2013.

[14] A. Dadgarpour; B. Zarghooni; B. S. Virdee; T. A. Denidni; A. A. Kishk, "Mutual Coupling Reduction in Dielectric Resonator Antennas Using Metasurface Shield for $60 \mathrm{GHz}$ MIMO Systems, " in IEEE Antennas and Wireless Propag. Lett., 2016 (in press).

[15] D. Guha and S. Biswas, "Characterization of Defected Ground Structure to be used Between Two DRA Array Elements for Suppressing the Mutual Coupling," in Proc. IEEE AP-S Int. Symp., Jul. 2012.

[16] Iqbal, J., U. Illahi, M. I. Sulaiman, M. Alam, and M. S. Mazliham. "Circularly Polarized Bandwidth Enhancement using Hollow Rectangular DRA." In Engineering Technology and Technopreneurship (ICE2T), International Conference on, pp- 1-4, 2017.

[17] Illahi, U., J. Iqbal, M. I. Sulaiman, M. Alam, and M. S. Mazliham. "A Novel Singly Fed Wideband Circularly Polarized Rectangular Multilayer DRA using Conformal Hook-Shaped Metal Strip," In Engineering Technology and Technopreneurship (ICE2T), International Conference, pp-1-4,2017.

[18] Payandehjoo, Kasra, and Ramesh Abhari. "Isolation Enhancement Between Tightly Spaced Compact Unidirectional Patch-Antennas on Multilayer EBG Surfaces." International Journal of RF and Microwave Computer-Aided Engineering, vol 25,no 1, pp-30-38, 2015.

[19] M.T. Lee, K.M. Luk, E.K.N. Yung, and K.W. Leung, "Microstrip Line Fed Circularly Polarized Rectangular Dielectric Resonator Antenna", Microwave and Optic. Tech. Lett. Vol 24, pp- 206-207, 2000.

[20] Sahu, Nikesh Kumar, Gourab Das, and Ravi Kumar Gangwar. "Dielectric Resonator-Based Wide Band Circularly Polarized MIMO Antenna with Pattern Diversity for WLAN Applications," Microwave and Optical Technology Letters, Vol 60, no. 12, pp. 2855-2862, 2018. 
[21] Das G, Sharma A, Gangwar RK. "Dielectric Resonator Based MIMO Antenna with Polarization Diversity". Microw. Opt. Technol. Lett. Vol 60, pp. 685-693, 2018.

[22] Sharma A, Das G, Ganwar RK. "Dual Polarized Triple Band Hybrid MIMO Cylindrical Dielectric Resonator Antenna for LTE2500/WLAN/ Wimax Applications". Int. J. RF Microw. comput. Aided Eng. Vol 26, pp. 763-772, 2016.

[23] Nayan MKA, Jamlos MF, Jamlos MA. "MIMO Circularly Polarized Array Antenna with Dual Coupled 900 Phased Shift for Point-to-Point Application". Microw. Opt. Technol. Lett. Vol 57, pp. 809-814, 2015.

[24] Sahu, Nikesh Kumar, Gourab Das, and Ravi Kumar Gangwar. "Dual Polarized Triple-Band Dielectric Resonator Based Hybrid MIMO Antenna for WLAN/Wimax Applications," Microwave and Optical Technology Letters. Vol 60, no. 4, pp. 1033-1041, 2018.

[25] Abedian, M., S. K. A. Rahim, S. Danesh, M. H. Jamaluddin, and M. T. Islam. "Compact Wideband Circularly Polarised Dielectric Resonator Antenna," Electronics Letters, vol 53, no. 1, pp. 5-6,2016.

[26] J. Iqbal, U. Illahi, M. I. Sulaiman, M. Alam and M. S. Mazliham, "Bandwidth Enhancement of Rectangular Dielectric Resonator Antenna with and Without a Parasitic Patch," Journal of Engineering Technology, vol 5,pp 5-8, 2017.

[27] Trivedi, Kedar, and Dhaval Pujara. "Suppression of Mutual Coupling in Wideband Tree Shaped Fractal DRA Array for MIMO Applications," In Antennas and Propagation (EUCAP), 2017 11th European Conference, IEEE, pp2209-2211, 2017.

[28] Malviya, Leeladhar, Rajib Kumar Panigrahi, and M. V. Kartikeyan. "MIMO Antennas with Diversity And Mutual Coupling Reduction Techniques: A Review." International Journal of Microwave and Wireless Technologies, vol 20, pp-1763-1780,2017. 